\title{
Role of Magnetic Resonance Cholangiopancreatography in Evaluation of Biliary Obstruction
}

\author{
Mohamed Farouk Aggag, Mohamed Said Abdul Aziz Shehata, Ziad El Sayed El Sayed Badawy \\ Department of Radiology, Faculty of Medicine, Al-Azhar University
}

Corresponding author: Ziad El Sayed El Sayed Badawy, Mobile: +201005307423,

E-Mail: ziadelsayed@ hotmail.com

\begin{abstract}
Background: obstructive jaundice which is caused by bile duct obstruction can be clinically and biochemically indistinguishable from cholestatic jaundice caused by hepatocellular disease. The management of both these conditions being radically different, the principle task of the radiologist is to differentiate between hepatocellular and obstructive jaundice, by using available imaging modality and help in further management. With the availability of non-invasive modality like magnetic resonance imaging (MRI), it is possible to diagnose obstructive jaundice early and accurately without any patient discomfort. The purpose of this article is to describe the protocol for evaluation of obstructive jaundice with use of magnetic resonance cholangio pancreatography sequence of MRI and to describe the imaging features of the most common causes of obstructive jaundice like biliary calculi, bile duct strictures, choledochal cyst, gall bladder carcinoma, cholangiocarcinoma, primary sclerosing cholangitis, and pancreatic head carcinoma. Aim of work: this work aimed to study and evaluate the role of MRCP in patients with biliary obstruction. Patients and Methods: this study included 30 patients with biliary obstruction during the period from December 2017 to October 2018. MRCP was performed on a 1.5 T MRI system, using a phased-array body coil. Fasting for 4 hours prior to the examination is required to reduce gastro-duodenal secretions, reduce motility to eliminate motion artifacts and to promote distension of gall bladder.For optimum visualization of ducts, acquired images were reformatted in different planes using multiplanar reconstruction (MPR) and maximum intensity projection (MIP). Results: in all cases, MRCP displayed the different parts of the biliary tract and localized the exact site of obstruction in all of the obstructed cases. According to the morphology encountered at the site of obstruction, MRCP was found highly specific in differentiating the calcular, malignant and benign causes of obstruction. The quality of images were degraded by the presence of massive ascites, marked patient obesity in our study by the instability to hold breath in a reproducible manner. Lack of the therapeutic role and the inability to perform functional studies mainly at the papilla remain the main limitation of the technique. Conclusion: MRCP should be the next step following depiction of biliary obstruction by U/S. Consequently in the near future there will be no place for the diagnostic use of the ERCP which shall be then restricted to a therapeutic role.
\end{abstract}

Keywords: MRCP, obstructive jaundice, biliary stricture, cholangiocarcinoma, choledocholithiasis

\section{Introduction:}

MRCP is now commonly used as a noninvasive diagnostic tool in visualization of the pancreaticobiliary tree without injection of contrast media, exposure to ionizing radiation or the injurious puncture of PTC ${ }^{(1)}$. It can be used to establish the presence and severity of biliary and pancreatic ductal dilatation, detect the length of the stricture as well as the exact cause and location of the obstructing lesion ${ }^{(2)}$. MRCP was first used in the 1990s. Since then it has undergone considerable technical evolution and maturation and has reached the stage at which MRCP has become an alternative to diagnostic endoscopic

retrograde cholangiopancreatography $(\mathrm{ERCP})^{(3)}$.

MRCP is based on heavily T2 weighted sequence that presents static or slowly moving fluids including bile as bright (hyperintense) structures against a dark (hypointense) back ground of surrounding organs. The initial MRCP studies were performed with gradient ECHO sequences by using $2 \mathrm{D}$ or $3 \mathrm{D}$ steady state free prescession sequence, however a limitation to such sequences was their inability to display non dilated bile ducts, recent 
developments include fast high- spatialresolution MRCP sequences such as heavily T2 weighted turbo spin echo (TSE), Single shot rapid acquisition with relaxation enhancement (RARE) and half Fourier single shot TSE (HASTE). These new modes of MR sequencing provide projection images that are similar to images obtained using ERCP. Therefore to conclude the higher the field strength and the shorter the scanning time, the better the image quality because of higher signal-to-noise ratio ${ }^{(4)}$.

\section{Patients and Methods:}

This study included 30 patients with biliary obstruction during the period from December 2017 to October 2018. MRCP was performed on a $1.5 \mathrm{~T}$ MRI system, using a phased-array body coil. Fasting for 4 hours prior to the examination is required to reduce gastroduodenal secretions, reduce motility to eliminate motion artifacts and to promote distension of gall bladder.For optimum visualization of ducts, acquired images were reformatted in different planes using multiplanar reconstruction (MPR) and maximum intensity projection (MIP). The study was approved by the Ethics Board of Al-Azhar University.
Obtained MRI sequences were:

- RARE: rapid acquisition and relaxation enhancement

- FRFSE: fast-recovery fast spin-echo coronal oblique 3D respiratory triggered

- HASTE: half-Fourier acquisition single shot turbo spin echo-axial 2D breath hold sequence which provide superior images and can be performed in single breath hold $(<20 \mathrm{~s})$ and a fatsuppressed sequence

- Axial and coronal T2 sequences.

- Additional fat suppressed T1 GRE sequence was acquired to evaluate duct wall.

- The advantage of FRFSE, as a 3D technique, was the ability to perform multiplanar reconstructions.

The average duration time of the examination was from 25 minutes up to 30 minutes.

\section{Results:}

The mean age in the studied patients was $52.17 \pm 10.5$ years with minimum age of 30 years and maximum age of 69 years.

Table 1: description of age in the studied patients

\begin{tabular}{|c|c|c|}
\hline \multicolumn{2}{|l|}{ Variable } & \multirow{2}{*}{$\begin{array}{c}\text { Studied patients } \mathbf{N}=(\mathbf{3 0}) \\
52.17\end{array}$} \\
\hline \multirow{4}{*}{$\begin{array}{c}\text { Age } \\
\text { (years) }\end{array}$} & Mean & \\
\hline & $\pm \mathrm{SD}$ & 10.5 \\
\hline & Min & 30 \\
\hline & Max & 69 \\
\hline
\end{tabular}

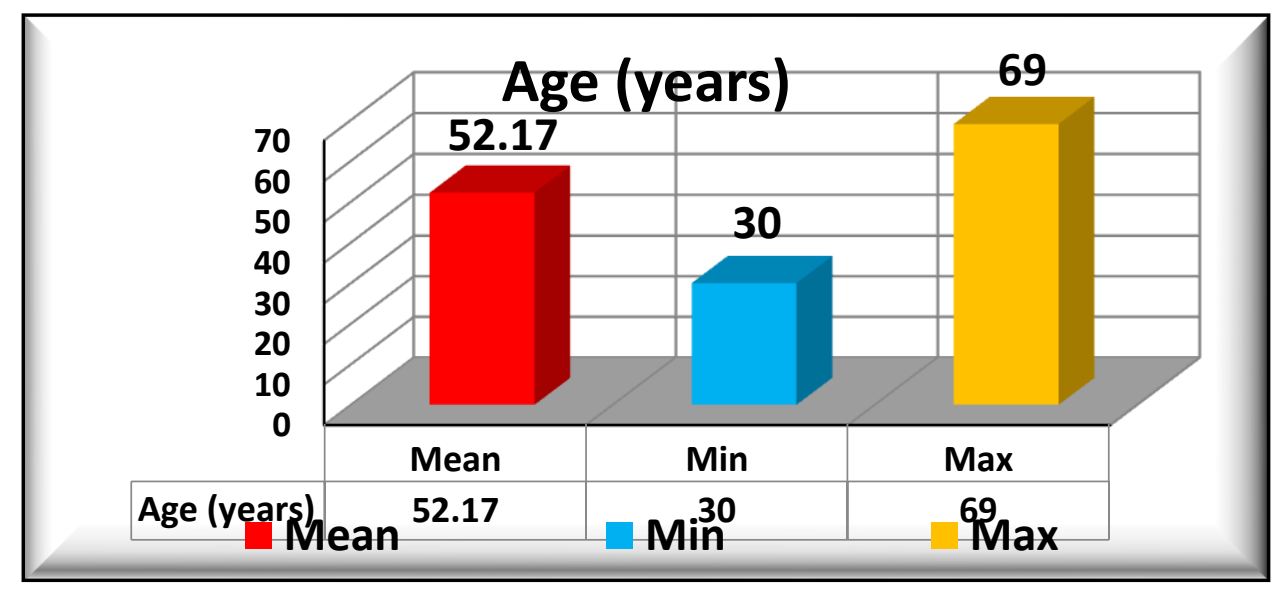

Fig.1: description of age in studied patients 
We noticed that 12 patients $(40 \%)$ were calcular, 5 patients (16.7\%) were inflammatory, 8 patients (26.7\%) were neoplastic, 4 patients (10\%) were post-operative and 2 patients $(6.7 \%)$ were miscellaneous.

The examinations were preceded by conventional axial \& coronal sections FSE T2WI which were used as a guide to optimally localize the biliary tract and to properly plan the coronal MRCP slabs.

Table 2: description of etiology in studied patients

\begin{tabular}{|c|c|c|}
\hline \multicolumn{2}{|l|}{ Variable } & Studied patients $N=(30)$ \\
\hline \multirow{5}{*}{ Etiology } & Stone & $12(40 \%)$ \\
\hline & Inflammatory & $5(16.7 \%)$ \\
\hline & Neoplastic & $8(26.7 \%)$ \\
\hline & Post-operative & $3(10 \%)$ \\
\hline & Miscellaneous & $2(6.7 \%)$ \\
\hline
\end{tabular}

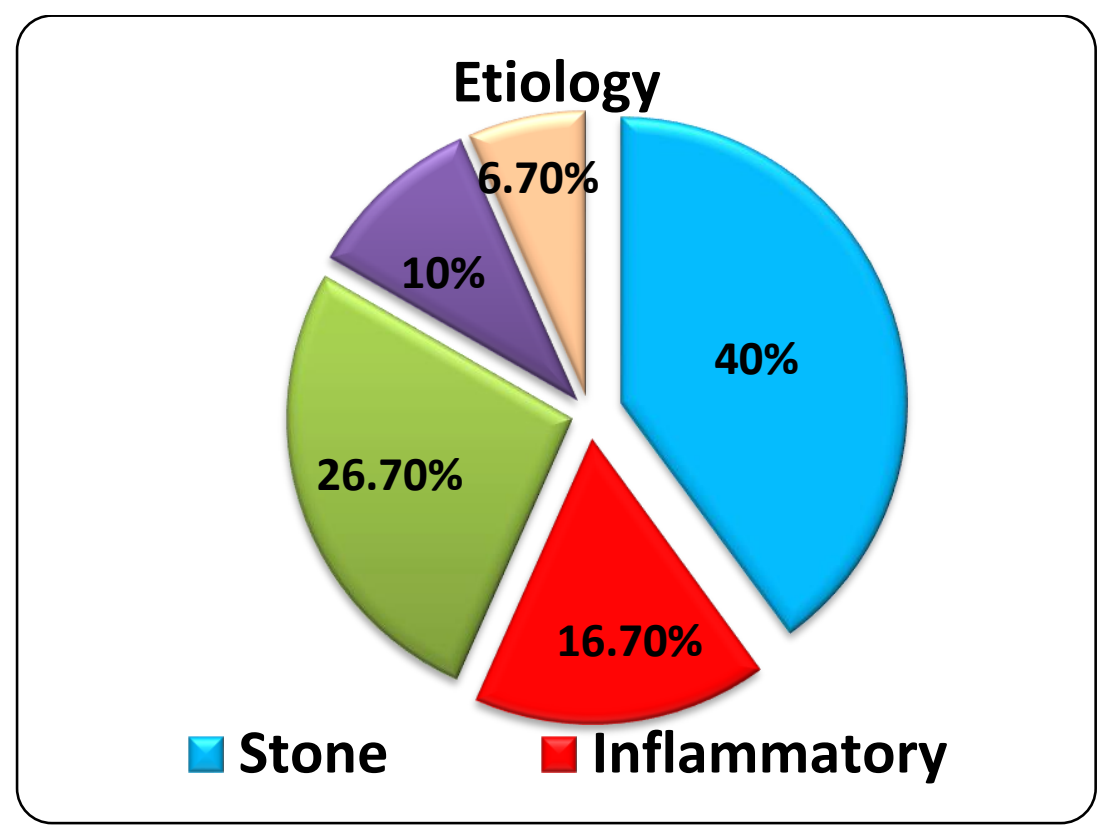

Fig.2: description of etiology in studied patients

Moreover, the provided cross sectional diagnostic information assessed the MR cholangiograms in confirming or excluding certain diagnosis and occasionally helped in staging neoplastic lesions. In all the examined cases, we commented on the visualization \& degree of dilatation of the different parts of the bilio-pancreatic tree, level of obstruction if present, the morphological aspect at the obstruction site $\&$ any additional information provided by the cross sectional images. Finally, we set a conclusion guided by the aforementioned data which was correlated with the ultrasound findings and/or histopathological or surgical specimen.

\section{Discussion}

The

emergence of MR cholangiopancreatography (MRCP) is considered as one of the most rapid success stories in recent memory since first reported in 1990. Before this date the non invasive diagnosis of biliary obstruction relied mainly on ultrasound (US) and computed tomography (CT), whose accuracy was limited mainly by their low sensitivity in diagnosis of CBD stones $\quad{ }^{(5)}$ Endoscopic retrograde cholangiopancreatography (ERCP) had a therapeutic role allowing sphincterotomy, stone removal, exfoliative cytology and/or stent placement was therefore often required. It presented a success rate varying from $70 \%$ $90 \%$ which increased with operator 
experience. ERCP, however was an invasive procedure with a morbidity rate of $1 \%-7 \%$ and mortality rate of $0.2 \%-1 \%{ }^{(6)}$. MRCP has developed as a non invasive technique permitting volume image acquisition that depicts bile and pancreatic ducts in a manner analogous to that of familiar radiographic films. This type of ductal imaging has become a part of comprehensive cross sectional MR imaging for identification of various pathological processes of the pancreaticobiliary system ${ }^{(7)}$. MRCP was based on heavily T2 weighted sequence that allowed imaging static or slowly flowing fluids with long T2 relaxation times, including bile and pancreatic secretions. They appeared as high signal intensity, high contrast, bright structures against a dark background of parenchymal and adipose tissue with a very low signal intensity ${ }^{(8)}$. The obtained images when displayed and reconstructed by maximum intensity projection (MIP), the appearance closely resembled that derived from cholangiography (ERCP or PTC). However, this was achieved in a non invasive manner without the need for exposure to ionizing radiation or contrast medium administration either directly or intravenously. The aim of the present study was to evaluate the role of MRCP as a rather rapid developing non invasive technique in the evaluation of different causes of biliopancreatic tract obstruction. We tried to assess its sensitivity in detecting biliary obstruction and specificity in determining the nature of the obstructive lesion. We also tried to demonstrate its advantages and highlight its disadvantages and limitations. Accordingly the two major elements of the tale were the imaging radiological techniques and the clinical impact. MRCP had evolved and rapidly progressed as a result of interactions between the advances in fast MR imaging techniques specially the fast spin echo (FSE) sequences, image post processing such as maximum intensity projection (MIP) and various techniques for suppression of respiratory motion artifacts. The latter being the major obstacle to MRCP as any MR examination of the abdomen and accordingly, MRCP techniques can be broadly classified into breath hold and non breath hold techniques. The non breath hold techniques had expanded the utility of MRCP as most patients were elderly, uncooperative and could not hold their breath for 20-40 seconds as required by most breath hold techniques. Respiratory triggering was the most reliable method for reducing respiratory motion artifacts in non breath hold MRCP performed during quiet breathing by averaging multiple signals. Using a belt to monitor respiratory movement, the scan was triggered at end expiration with the parameters modified to ensure that scanning is completed before the next inspiratory cycle. These results showed moderate (30-50\%) decrease in the scanning efficiency and prolongation of the scan time because no scanning was performed during inspiration. However this is compensated for by the excellent suppression of breathing artifacts \& the decreased need of patients cooperation which agrees with the results of Graham et $\boldsymbol{a l} \boldsymbol{l}^{\left({ }^{(9)}\right.}$. Respiratory triggered MRCP could be achieved both by 2D and 3D FSE sequences. The major limitations of all 2D FSE approaches was that the slices acquired were relatively thick $(3 \mathrm{~mm})$, limiting the resolution of the image and therefore the minimum size of a structure that can be detected. In addition, the anisotropic resolution with slice thickness was more twice than in plane resolution degrades the quality of MIP or multiplanar reformation. Therefore oblique slices parallel to the duct of interest or as many as three separate planes may need to be prescribed. The 3D FSE approach did not lend itself to more than one or two averages, so respiratory triggering was used to suppress motion artifacts. However, the use of 3D multiple slab imaging allows section thickness to be reduced to $2 \mathrm{~mm}$ with an adequate signal to noise ratio. Consequently the smaller ratio of slice thickness to in plane resolution compared with the 2D FSE technique allowed reformatting or MIP to be performed in virtually any desired plane with minimal sacrifice of image quality. Therefore, for most applications, a single coronal acquisition sufficed for most patients which agrees with the results of Zhang $\boldsymbol{e t}$ al ${ }^{(\mathbf{1 0})}$.

Recently two fast (single shot) breath hold techniques had been applied. The single shot rapid acquisition by relaxation enhancement (RARE) and the half Fourier acquisition single shot turbo spin echo (HASTE). Both allowed breath hold imaging 
in less than 20 seconds, albeit with relatively low in-plane resolution. In the single shot RARE, each ECHO train compresses the full set of phase encoding steps as determined by the matrix size whereas, the use of HASTE reduced the number of phase encoding steps by as much as $40 \%$ while, maintaining the same spatial resolution. Because these techniques use a linear profile K-space ordering, effective ECHO times on the order of $1000 \mathrm{msec}$. can be obtained with the current ranges of minimum echo spacing available. These techniques interrogate one section of a tissue at a time, they are implemented in either single-slice mode or sequential multiple slice mode ${ }^{(11)}$. In the multiple slice mode, several slices (typically 4-5 $\mathrm{mm}$ thickness) are obtained, with the total number of slices limited by the duration of the breath-hold time. Post processing with MIP algorithm is then performed to create a 3D image so that signals from moderate/large ducts are enhanced (12). However, with MIP much of the information contained on individual source images can be lost when a whole volume is displayed. Although small structures and abnormalities were depicted on source images, they can be missed on MIP images. Therefore relying on individual source images for interpretation is critical. In addition, because of the sequential acquisition of 2D images, miss registration in patients who could not hold their breath for at least 20 seconds and consequently the spatial resolution in the slice selection direction were significantly inferior to that in either of the other two directions. In the thick signal slice mode, (20-120 mm) multiple acquisitions in several oblique planes were necessary to obtain all necessary diagnostic views. This method did not generate a 3D data set suitable for post processing or creation of MIP images. This MRCP technique provided the most direct method to maximally shorten the acquisition time with consequent avoidance of the respiratory misregistration and fast intestinal movements. It required no post processing apart from image reconstruction which can be completed in less than 3 seconds. In the present study all the cases where examined using this breath hold HASTE sequence " single slice technique". In this sequence, two significant features where implemented. A FSE sequence allowed a large number of ECHOs and conjugated K-space filling that speeds up the data acquisition taking only 3-5 seconds which rendered the technique feasible even in elderly or suffering patients. There was no need for the time-consuming post processing procedures. We found that a slice thickness ranging between 40 and $60 \mathrm{~mm}$ are usually adequate to include the major portions of the bilio-pancreatic tract which was the same conclusion reached by Lee et al $^{(\mathbf{1 2 )}}$. The referring surgeons and endoscopists were familiar with the obtained images as closely resembling the appearances; they were used to direct cholangiography ( PTC, ERCP). However, the main potential disadvantages encountered during the study were occasional veiling of small gall bladder calculi by the high signal from fluid in the gall bladder owing to the partial volume effects. These small calculi were only depicted on complementary axial T2WI routinely done in every examination. Moreover a variable degree of blurring artifact was noted especially at large projection thickness The obtained image quality by this technique was influenced by many factors. Massive ascites was found a factor degrading the image quality as the long T2 relaxation time of the ascitic fluid increases the signal of the background with consequent decrease in the contrast between it and the biliary tree which was also stated by Polakova et al. ${ }^{(13)}$. Marked patient obesity was also found a factor that partially degraded the image quality causing significant drop in the signal to noise ratio. This agrees with the statement of Uppot et al. ${ }^{(14)}$

Among the 30 cases done in our study, MRCP has accurately localized the site of obstruction in all the obstructed cases (100\%). This agrees with results of Kurtul $\boldsymbol{e t}$ al. that stated $100 \%$ sensitivity of MRCP in detecting biliary obstruction ${ }^{(5)}$.

In most of the cases with proximal obstruction sites, they demonstrated the collapsed biliary tract distally. In the calcular group, intraluminal filling defect was the cardinal sign in diagnosing calcular obstruction being encountered in 12 cases (40\%). MRCP was able to diagnose choledocal calcular obstruction in all of the 12 cases. Of those only 4 (33\%) had calcular gall bladders, while 2 cases $(16 \%)$ 
had their gall bladder removed. The technique presented $97 \%$ specificity in determining calcular obstruction with only a single missed case that presented poor quality of the images. This agrees with the results of Kurtul $\boldsymbol{e t} \quad \boldsymbol{a l}$. who reported a $96 \%$ specificity of MRCP in determining choledocal calcular obstruction ${ }^{(5)}$. In the malignant cases, the abrupt cut off termination was the commonest finding encountered in 6 Cases, while missed segment of biliary tract and short eccenteric stricture were encountered in 2 Cases.

The technique was accurate in determining the malignant nature of obstruction in $94 \%$ of cases compared to an accuracy of $71 \quad \% \quad$ in the study of Parashari et al $^{\left({ }^{(6)}\right.}, 96.3 \%$ by Suthar $\boldsymbol{e t} \boldsymbol{a l} .{ }^{(7)}$ and $100 \%$ by kurtul $\boldsymbol{e t}$ $\boldsymbol{a l}{ }^{(5)}$.Although these rates were higher than those in the literature, the low number of malignant cases should be considered. Also, in this group the axial T2WI showed hepatic metastatic deposits in 2 cases, changing them to stage IV and decided unressectability of the primary tumour. The technique presented comparable specificity of $97 \%$ \& $93.8 \%$ in calcular and malignant cases respectively regarding its ability to decide the nature of obstructive lesion. In the inflammatory group, central and extrahepatic duct dilatation was typically seen with abrupt tapering of peripheral ducts in 5 cases (16\%). Of those 4 cases had post cholecystectomy recurrent cholangitis, while 1 case had acalcular cholecystitis. The technique was accurate in determining the inflammatory nature of obstruction in 100 $\%$ of cases compared to an accuracy of $98 \%$ in the study of Park et al. ${ }^{(8) .}$

The fourth group was constituted by post cholecystectomy patients. Because of its capability to provide non-invasive high-quality visualization of the biliary tract, magnetic resonance cholangiopancreatography (MRCP) showed a sensitivity of $100 \%$ \& a specificity of $67 \%$ compared with Schofer JM 2008 which stated that MRCP has a sensitivity of $95-100 \%$ and a specificity of $88-89 \%$ for detecting post cholecystectomy biliary obstruction causes. ${ }^{(5)}$ The fifth group was constituted of primary sclerosing cholangitis patients, where MRCP display of the biliary tract, the hepatic parenchyma and perihepatic lymph nodes greatly helped solidify the diagnosis and served as a road map if endoscopic interventional procedures are anticipated. The results have shown that MRCP had a sensitivity of $100 \%$ and a specificity of $90 \%$ in comparison with Fulcher et al. ${ }^{(15)}$ and Berstad $\boldsymbol{e t}$ al. ${ }^{(16)}$ studies who collectively gave a sensitivity range of $80 \%$ to $88 \%$ and a specificity range from $87 \%$ to $99 \%$. As we see, despite the high sensitivity of the MRCP technique in detecting biliary dilatation and obstruction site, it showed wide variability in its specificity to identify the nature of the obstructing lesion. To sum up, this study demonstrated the advantages of MRCP as a developing technique in evaluating patients with suspected biliary obstruction as follows:

It proved as a totally non invasive, safe, well tolerated and consequently repeatable procedure in patients presenting with poor symptoms. It did not require contrast medium administration or routine patient sedation and no complications were encountered in adequately screened patients. MRCP provided global representation of the biliary and pancreatic duct systems, both proximal and distal to the site of biliary obstruction in all the examined cases. Moreover, it allowed multiplanar views of the bilio-pancreatic tract without the need to mobilize the patient.According to the morphological pattern encountered at the site of obstruction, MRCP can depict the nature of obstructing lesion in a manner analogous to direct cholangiography. Intraluminal filling defect (s) and/or the meniscus sign were found the cardinal signs to diagnose calcular obstruction, whereas abrupt cut off termination, abrupt caliber change, missing segment or short eccentric stricture were the diagnostic signs of malignant obstruction.

MRCP can be applied in cases with technical limitations that can prevent successful ERCP. In our study, this was mainly in cases of previous biliary enteric anastomosis.

However, MRCP was not a technique without disadvantages; its main limitations can be confined to:

- The lack of therapeutic role when compared to the interventional 
procedures allowed by ERCP and to lesser extent PTC.

- MRCP doesn't enable functional studies permitted by ERCP at the papilla.

- Peri-ampullary lesions and anomalies remain a challenge for MRCP where ERCP is much more superior by allowing direct endoscopic view and permitting biopsy of any suspicious abnormality.

- Degradation of the obtained image quality by many factors including the patients obesity and the presence of massive ascites. Moreover, in breath hold techniques (as in our study), the inability of the patient to hold his breath in a reproducible fashion, only during data acquisition, was a marked limiting factor, encountered only in one case during the present study.

\section{Conclusion:}

MRCP will in the recent future modify the approach to patients presenting with biliary obstruction that is because it can totally replace the diagnostic role of ERCP and the staging role of $\mathrm{CT}$ in malignant biliary obstruction. Thus, distinguishing between operative lesions and those only requiring palliative treatment in a completely non invasive manner

One can recommend that MRCP should be performed as the next step after depicting biliary obstruction by US. MRCP will provide the basis for determining not only the subsequent diagnostic protocol but also the suitable therapeutic procedure whether surgical or interventional.

Finally, considering the predominant role of ERCP in this pathology, both diagnostic and therapeutic, it is likely that there will be changes in ERCP indications, restricting the diagnostic ones but totally maintaining the therapeutic applications.

\section{References}

1. Shanbhogue AKP, Tirumani SH, PrasadSR, Fasih Nand McInnes M(2011): B iliary Strictures, A Current Comprehensive Clinical and Imaging Review. AJR., 197:W295-306.
2. Halefoglu AM (2007): Magnetic resonance cholangiopancreatography, a useful tool in the evaluation of pancreatic and biliary disorders. World J .Gastroenterol., 13: 25292534.

3. Fernández-Esparrach G, Ginès A, Sánchez M et al. (2007): Comparison of endoscopic ultrasonography and magnetic resonance cholangiopancreatography in the diagnosis of pancreatobiliary diseases, a prospective study. Am. J .Gastroenterol., 102:1632-1639.

4. Katabathina VS, Dasyam AK, Dasy am N, Hosseinzadeh K et al.(2014): Adult bile duct stricture, role of MRI and Magnetic resonance cholangiopancreatography in characterization. Radio. Graphics, 34(3):565586.

5. Kurtul Yıldız H, Şahin G, Ekin EE, Erok B and Adaş GT (2017):Diagnostic contribution of magnetic resonance cholangiopancreatography in biliary obstruction, additional findings and misdiagnosis. JAREM. , 7: 135-149.

6. Parashari UC, Khanduri S, Bhadur y S et al. (2015): Diagnostic role of magnetic resonance cholangiopancreatography in evaluation of obstructive biliopathies and correlating it with final diagnosis and clinical profile of patients. J. Nat. Sc. Biol. Med., 6: 131-138.

7. Suthar M, Purohit S, Bhargav V

and Goyal $P$ (2015): Role of MRCP in differentiation of benign and malignant causes of biliary obstruction. J .Clin. Diagn. Res ., 9: $8-12$.

8. Park MS, Yu JS, Lee JH and Kim KW (2007): Value of manganese-enhanced T1- and T2-weighted MR cholangiography for differentiating cystic parenchymal lesions from cystic abnormalities which communicate with bile ducts. Yonsei. Med. J., 48:10721074.

9. Graham C et al. (2004): Magnetic resonance cholangiopancreatographyevaluation of image quality in breath-hold and non-breath-hold technique.

Radiography, 10(3):195 - 199.

10. Zhang J, Israel GM, Hecht EM, Krinsky GA, Babb JS and Lee VS (2006): Isotropic 3D T2 -weighted MR cholangiopancreatography with parallel imaging: feasibility study. AJR. Am. J. Roentgenol., 187:1564-1570. 
11. Wallnoefer AM, Herrmann KA, Beuers U et al. (2005): Comparison of 2D and 3D sequences for MRCP, Clinical value of the different techniques. Radiologe, 45(11): 9961003.

12. Lee MG, Jeong YK, Kim MH et al. (1998): MR cholangiopancreatography of pancreaticobiliary diseases: comparing singleshot RARE and multislice HASTE sequences. AJR., 171:1539-1545.

13. Polakova K1, Mocikova $P$, Tucek $P$ et al. (2016): Magnetic resonance cholangiopancreatography (MRCP) using new negative per-oral contrast agent based on superparamagnetic iron oxide nanoparticles for extrahepatic biliary duct visualization in liver cirrhosis. Biomed. Pap. Med. Fac. Univ. Palacky Olomouc Czech. , 160(4):512-517.
14. Uppot RN, Sheehan A and Seethamraju R(2005): MRI hot topics: besity and MR imaging.American J. of Radiology, 188(2):433-440.

15. Berstad AE, Aabakken L,SmithH J, Aasen S, Boberg KM and Schrumpf E(2006): Diagnostic accuracy of magnetic resonance and endoscopic retrograde cholangiography inprimary sclerosi ng cholangitis. Clin. Gastroenterol. Hepatol. 4:514-520.

16. Fulcher AS, Turner MA, Capps GW, Zfass AM and Baker KM(1998): HalfFourier RARE MR cholangiopancreatography: experience in 300 subjects. Radiology, 207(1):21-32. 